\title{
Operación de Karydakis ambulatoria en el manejo de la enfermedad pilonidal sacrococcígea*
}

\author{
Drs. GUILLERMO BANNURA C. ${ }^{1}$, ALEJANDRO BARRERA E. ${ }^{1}$, \\ JAIME CONTRERAS P. ${ }^{1}$, CARLOS MELO L. ${ }^{1}$, DANIEL SOTO C. ${ }^{1}$
}

\begin{abstract}
1 Servicio y Departamento de Cirugía Hospital Clínico San Borja Arriarán, Campus Centro, Facultad de Medicina Universidad de Chile. Santiago, Chile.
\end{abstract}

\begin{abstract}
Ambulatory Karydakis procedure for sacrococcygeal pilonidal disease

Background: Asymmetrical closed techniques have comparative benefits for the treatment of sacrococcygeal pilonidal disease. Aim: To assess the results of Karydakis asymmetrical excision, performed on an ambulatory basis. Patients and Methods: Prospective evaluation of 29 females and 21 males aged 15 to 44 years operated using Karydakis procedure on an ambulatory basis. Results: The body mass index of patients fluctuated between 19 and $47 \mathrm{~kg} / \mathrm{m}^{2}$. Thirty eight percent were overweight and $28 \%$ were obese. Sixty two percent were hirsute. The form of presentation of the disease was the spontaneous or surgical drainage of an abscess with chronic suppuration in $92 \%$ of cases. Surgical time fluctuated between 25 and 45 minutes. All patients were discharged four to six hours after the procedure. There were no re-operations and the rate of complications was $10 \%$. Ninety percent of patients did not have a local complications and a complete healing was achieved three weeks after the operation. After a follow up of 3 to 65 months, no relapses have been observed. Conclusions: Karydakis procedure, performed on an ambulatory basis, is safe, feasible and has excellent results.
\end{abstract}

Key words: Karydakis procedure, pylonidal disease, ambulatory surgery.

\section{Resumen}

Antecedentes: Las técnicas cerradas asimétricas tienen ventajas comparativas en el manejo de la enfermedad pilonidal sacrococcígea (EPSC). El objetivo de este estudio es evaluar los resultados de la resección asimétrica de Karydakis programada como cirugía mayor ambulatoria. Pacientes y Método: Estudio prospectivo de 100 pacientes intervenidos en forma electiva desde abril de 2001; 84 con la técnica de Karydakis y, de ellos, 50 pacientes en forma ambulatoria. Se utilizó la técnica clásica descrita por el autor, con pequeñas modificaciones que intentan alejar la cicatriz de la línea media. Resultados: Se trata de 29 mujeres y 21 hombres, con un promedio de edad de 21,2 años (extremos 15-44). El IMC promedio fue de 27,28 (extremos 19-47), sin diferencias estadísticas según el género. El 38\% de los pacientes tenían sobrepeso y el $28 \%$ eran obesos. El $62 \%$ de los pacientes fue catalogado como hirsutos, con claro predominio de los pacientes varones $(\mathrm{p}=0,012)$. La forma clínica de presentación fue el drenaje espontáneo o quirúrgico de

*Recibido el 2 de Diciembre de 2008 y aceptado para publicación el 12 de Enero de 2009.

Correspondencia: Dr. Guillermo Bannura C.

Las Limas 1622, Santiago, Chile.

E-mail: gbannura@vtr.net 
un absceso con supuración crónica intermitente en el $92 \%$ de los casos. El tiempo operatorio promedio de 34 minutos (extremos 25-45). Todos los pacientes fueron dados de alta entre 4 y 6 horas luego del procedimiento, no hubo reoperaciones y la morbilidad global fue $10 \%$. El $90 \%$ de los pacientes no tuvo complicaciones locales logrando una cicatrización completa antes de 3 semanas de la intervención. El seguimiento promedio de la serie es 30 meses (extremos 3-65) sin casos de recidiva $(1,19 \%$ - 1/84) en la serie global con un período de observación promedio de 49 meses (extremos 6-88). Conclusión: La operación de Karydakis realizada en forma ambulatoria es factible, segura y muestra excelentes resultados en tiempo de cicatrización, morbilidad y recidiva. Se propone como la técnica de elección en el manejo de la mayoría de los casos de EPSC.

Palabras clave: Operación de Karydakis, quiste sacrocoxígeo.

\section{Introducción}

La enfermedad pilonidal sacrococcígea (EPSC) es una condición adquirida cuya fisiopatología aún no es completamente comprendida ${ }^{1-3}$. Se ha propuesto una gran variedad de procedimientos quirúrgicos para su manejo, sin que hasta la fecha exista consenso. Sin embargo, de la revisión de la literatura publicada en los últimos años se desprenden dos hechos relevantes: 1) Las técnicas cerradas tienen ventajas sobre las técnicas abiertas dado el prolongado período de cicatrización e incapacidad laboral de esta últimas; y 2) Dentro de las técnicas cerradas, las resecciones asimétricas (se incluye la técnica de Karydakis y variados tipos de plastías con rotación de colgajo) logran los mejores resultados en tiempo de cicatrización, reintegro laboral y tasas de recidiva ${ }^{4-7}$.

En el tratamiento de la EPSC la técnica de Kakydakis es particularmente atractiva por lo simple y reproducible, deja una cicatriz relativamente pequeña, no requiere de grandes incisiones ni desplazamientos de colgajo y exhibe tasas de recidiva entre 0 y $4 \%^{8-10}$. Inicialmente en las series publicadas con la operación de Karydakis se registra un tiempo de hospitalización de 2 a 7 días, evolucionando en el tiempo hacia internaciones cada vez más acortadas (en nuestra experiencia promedio 1,3 días) ${ }^{10}$ hasta llegar a la realización ambulatoria del procedimiento en la gran mayoría de los casos ${ }^{11}$. La factibilidad de efectuarla en forma ambulatoria reduce costos, mejora la aceptación del paciente al procedimiento y hace la intervención más atractiva en la búsqueda de una técnica ideal para el manejo de la EPSC ${ }^{4,5,11}$. El objetivo de este estudio es analizar los resultados inmediatos y alejados del colgajo de Karydakis programado como procedimiento ambulatorio.

\section{Material y Método}

En mayo de 2001 iniciamos un protocolo prospectivo de manejo de la EPSC electiva que contem- pla la resección asimétrica de Karydakis para los pacientes habituales (con la modificación de Kitchen $^{9}$ si existe un orificio fistuloso alejado de la línea media) y el colgajo de Limberg en los pacientes recidivados $\mathrm{y} / \mathrm{o}$ con múltiples orificios fistu$\operatorname{losos}^{10}$. Desde esa fecha se han intervenido 100 pacientes por EPSC en forma electiva, 10 mediante la plastía de Limberg, 6 mediante cierre primario simétrico (en los inicios del protocolo) y 84 mediante la técnica de Karydakis. Una vez adquirida mayor experiencia con el procedimiento, los pacientes fueron programados para cirugía ambulatoria con dicha técnica y forman el universo de este estudio. Todos los pacientes dieron el consentimiento informado para la intervención. Los criterios de inclusión son EPSC en paciente ASA I-II con una red de apoyo social compatible con cirugía mayor ambulatoria. Se excluyen los pacientes con infección aguda, los recidivados y aquellos con múltiples orificios fístulosos. No se utilizó preparación mecánica del colon. La anestesia fue espinal en todos los casos y como profilaxis se indicó una dosis de cloxacilina 1 gramo ev.

La técnica quirúrgica empleada corresponde al procedimiento clásico propuesto por el autor ${ }^{8} \mathrm{y}$ fue descrita en detalle previamente ${ }^{10}$. Algunos aspectos destacados que pueden ser relevantes en los resultados se mencionan brevemente: 1) Se traza una paramediana vertical a $2 \mathrm{~cm}$ de la línea media desplazada hacia el lado del orificio fistuloso o de la cicatriz y se talla una elipse que incluye toda la lesión; 2) El extremo caudal de la incisión es desplazada $1 \mathrm{~cm}$ más hacia lateral quedando a $3 \mathrm{~cm}$ de la línea media, de modo de alejar la herida del ano; 3) Luego de efectuada la resección con bisturí frío y efectuada la hemostasia se suelta el vendaje en $T$ de los glúteos, se talla un colgajo graso de $2 \mathrm{~cm}$ de profundidad que se sutura en la línea media con puntos separados de Vicryl 3/0, lo que aplana la fosita natal; 4) Una segunda capa de puntos separados de Vicryl 3/0 entre el borde libre del colgajo medial y el borde lateral de la herida cierra el espacio muerto; 5) Hemostasia meticulosa. Habitualmente no requiere drenaje. Cierre de la piel sin tensión 
con monofilamento 3/0 lo que permite apreciar una herida vertical ligeramente oblicua en posición lateral a la línea media; 6) Desde el traslado a su cama el paciente adopta la posición en decúbito supino, lo que, junto al vendaje compresivo, favorece la hemostasia en las primeras horas del postoperatorio.

Se define el sobrepeso u obesidad según el índice de masa corporal (IMC), considerando sobrepeso un IMC entre 25 y 29,9 y obesidad por sobre 30 . El hirsutismo se definió como variable dicotómica de acuerdo al criterio subjetivo del cirujano ${ }^{12}$. Se registra la morbilidad postoperatoria, considerando que una herida ha cicatrizado si no hay descarga de material serohemático o pus, separación de los bordes de la herida o eritema. En ausencia de signos de infección se retiran los puntos entre los 12 y 14 días y se permite una actividad física normal desde el mes de la intervención, con la recomendación de no hacer ciclismo o cabalgar por un período no inferior a 3 meses. Se define como recidiva la descarga de material purulento o serohemático que se prolonga por más de 3 meses o que aparece en cualquier momento de la evolución de una herida ya cicatrizada. El seguimiento se efectuó mediante controles en el policlínico de la especialidad y/o entrevistas telefónicas.

\section{Resultados}

Desde abril de 2003 se han intervenido con la técnica de Karydakis en forma ambulatoria 50 pacientes consecutivos, 29 mujeres y 21 hombres, con un promedio de edad de 21,2 (extremos 15-44; desviación estándar (DE): 10,78). El IMC promedio fue de 27,28 (extremos 19-47), sin diferencias estadísticas según el género. El $38 \%$ de los pacientes tenían sobrepeso y el $28 \%$ eran obesos. El $62 \%$ de los pacientes fue catalogado como hirsutos, con claro predominio de los pacientes varones $(\mathrm{p}=$ 0,012). Descontando la obesidad, el $12 \%$ de los pacientes tenía una o más enfermedades asociadas, destacando el tabaquismo en el $8 \%$ de los casos. De acuerdo al protocolo, no se intervino ningún paciente con una o más recidivas luego de una operación electiva. La forma clínica de presentación fue el drenaje espontáneo o quirúrgico de un absceso con supuración crónica intermitente en el $92 \%$ de los casos y el aumento de volumen sensible en la región sacrococcígea en el resto. El tiempo de evolución de los síntomas fluctuó entre 1 y 60 meses.

Se utilizó la resección asimétrica clásica descrita por Karydakis en todos los casos con un tiempo operatorio promedio de 34 minutos (extremos 2545). Todos los pacientes fueron dados de alta entre 4 y 6 horas luego del procedimiento, con un rein- greso debido a un sangrado postoperatorio que requirió observación por dos días (mujer de 23 años portadora de un síndrome hemorragíparo). No hubo reoperaciones y la morbilidad global fue $10 \%$. Hubo 3 casos de infección superficial que se manejan con curaciones locales, logrando la cicatrización definitiva a las 3 semanas y 2 casos de absceso con dehiscencia parcial de la herida y retardo de cicatrización de 2 y 3 meses, respectivamente. En 4 de los 5 pacientes, la herida operatoria no quedó bien lateralizada y el extremo distal se curvaba hacia el ano. La dehiscencia parcial se produjo en todos los casos en la porción más caudal de la herida operatoria. En forma global, el $90 \%$ de los pacientes no tuvo complicaciones locales logrando una cicatrización completa antes de las 3 semanas de la intervención. El seguimiento promedio de la serie es 30 meses (extremos 3-65) y el 50\% de los casos supera los 36 meses de control. Hasta el corte de este estudio no hay casos de recidiva.

\section{Discusión}

Aunque en esta serie hay un ligero predominio del sexo femenino $(1,4: 1)$, es claramente inferior a las antiguas series nacionales en las cuales la relación mujer/hombre era $3: 1^{13-15}$. Al considerar la serie global de 100 pacientes en este período, la relación es exactamente $1: 1$, lo que contrasta con las series anglosajonas que muestran sistemáticamente un predominio del sexo masculino de $3: 1$, proporción que se eleva a 17:1 en los países mediterráneos $^{3-6,16-18}$. Diferencias raciales en aspectos constitucionales como la profundidad del surco ínter glúteo, la obesidad y el hirsutismo se han invocado como posibles causas de esta distribución por género tan sorprendente en nuestro medio, pero la evidencia no apoya esta teoría ${ }^{12,19}$. En esta serie el promedio del IMC es 27,28 , el $38 \%$ de los pacientes tiene un sobrepeso y el $28 \%$ es obeso, cifras similares a la población general, lo que confirma la poca relevancia de esta condición en la etiopatogenia de la $\mathrm{EPSC}^{12}$. La prevalencia del hirsutismo tampoco fue particularmente destacado en estos pacientes, hecho paradojal para una enfermedad que literalmente significa "nido de pelos", factor que ha sido muy poco estudiado ${ }^{20}$.

El 92\% de nuestros casos se intervino por presentar en algún momento de la evolución drenaje espontáneo o quirúrgico de un absceso con supuración intermitente, cifra que en la literatura extranjera promedia el $66 \%$ con un máximo de $75 \% \%^{3-6,18}$. La forma clínica de presentación en esta serie refleja probablemente lo tardío del tratamiento en nuestro medio y podría influir en los resultados del tra- 
tamiento quirúrgico, por ejemplo, si hay un predominio de pacientes intervenidos en la etapa de aumento de volumen sensible sin supuración, como lo muestran algunas series. En la experiencia de Mentes $^{21}$ sólo 61 de 493 (12,3\%) pacientes intervenidos durante el servicio militar refería el antecedente de drenaje en una o más oportunidades.

Aspectos técnicos relevantes de la intervención son la resección con bisturí frío, la hemostasia meticulosa y el evitar el espacio muerto, con lo cual se intenta prevenir la colección de líquido serohemático que puede infectarse y drenar en forma crónica, lo que el paciente vivencia como una verdadera recidiva. Aunque el drenaje ha sido un tema muy discutido ${ }^{18,22}$, creemos que en la gran mayoría de los casos no es necesario y ha sido la principal causa de la prolongada hospitalización que exhiben algunas series ${ }^{4-6,21}$. En una experiencia de cirugía ambulatoria, se recomienda colocar un drenaje aspirativo que se retira 4 horas después de la intervención al momento del alta, lo que nos parece poco útil $^{11}$. El vendaje compresivo y la postura en decúbito supino ${ }^{23}$ en el período postoperatorio inmediato colapsa el espacio muerto y favorece la hemostasia, sin que hayamos observado efectos adversos.

Es fundamental lograr una adecuada lateralización de la herida operatoria, la que en su extremo distal debe alejarse aún más de la línea media y del ano. Los casos de dehiscencia parcial que hemos observado ocurren precisamente en esa localización, por lo que se ha recomendado que la resección adopte una posición ligeramente "oblicua"21. Este hecho parece ser tan relevante que, para prevenir las fallas de las técnicas de rotación de colgajo, recientemente se ha propuesto la resección asimétrica modificada de la plastía de Limberg ${ }^{24,25}$, que desplaza lateralmente el extremo distal del colgajo.

En esta serie ambulatoria de la resección asimétrica de Karydakis la morbilidad global fue 10\%, similar a nuestra serie con una hospitalización acortada (promedio 1,3 días) y no hemos tenido recidiva con un seguimiento promedio satisfactorio. Esto confirma que la técnica es factible, segura y costoefectiva en el escenario ambulatorio, similar a lo publicado por otros autores ${ }^{11,24}$. Esta experiencia se ha realizado en una población de la región Metropolitana que se atiende en el servicio público, la mayoría de ellos con una baja protección desde el punto de vista socioeconómico, lo que realza la factibilidad del manejo ambulatorio de la EPSC habitual. De este modo reservamos la internación para los pacientes con enfermedades asociadas severas y los casos complejos que requieren técnicas de mayor envergadura y una observación de 24 a 48 horas ( $10 \%$ en nuestra experiencia).
En esta serie programada en forma ambulatoria no hemos tenido casos de recidiva con un seguimiento promedio de 30 meses, cifra que en nuestra serie global alcanza al 1,19\% (1/84), luego de un período de observación promedio de 49 meses (extremos 6-88), lo que confirma lo publicado por otros autores con la técnica ${ }^{4,5,7-11,16-18,21,22}$.

En conclusión, la resección asimétrica de Karydakis ambulatoria no afecta los buenos resultados que exhibe la técnica en términos de complicaciones, tiempo de cicatrización y recidiva, por lo que se recomienda como técnica de elección en la gran mayoría de los casos de EPSC. Para algunos autores esta intervención es segura y factible en los casos de recidiva ${ }^{2,18}$, reservando las técnicas de movilización de grandes colgajos para los casos de EPSC compleja con múltiples orificios fistulosos. De este modo la técnica de Karydakis se aproxima al ideal de intervención para el manejo de la EPSC: cicatrización rápida, no requiere hospitalización, baja morbilidad y mínima recurrencia ${ }^{6}$.

\section{Referencias}

1. Bascom J, Bascom T. Failed pilonidal surgery. Arch Surg 2002; 137: 1146-1150.

2. Eftaiha MS, Abcarian H, Weinstein M, Rosemberg I, Salvati EP. The dilemma of pilonidal disease. Dis Colon Rectum 1977; 20: 278-286.

3. Da Silva JH. Pilonidal cyst. Cause and treatment. Dis Colon Rectum 2000; 43: 1146-1156.

4. Chintapatla S, Safarini N, Kumar S, Haboubi. Sacrococcygeal pilonidal sinus: historical review, pathological insight and surgical options. Tech Coloproctol 2003; 7: 3-8.

5. Petersen S, Koch R, Stelzner S, Wendlant T-H, Ludwig K. Primary closure techniques in chronic pilonidal sinus. A survey of the results of different surgical approaches. Dis Colon Rectum 2002; 45: 1458-1467.

6. Allen-Mersh TG. Pilonidal sinus: finding the right tract for treatment. Br J Surg 1990; 77: 123-132.

7. Bannura G. ¿Cuál es el tratamiento de elección de la enfermedad pilonidal sacrococcígea? Rev Chil Cir 2003; 55: 92-96.

8. Karydakis GE. Easy and successful treatment of pilonidal sinus after explanation of it's causative process. Aust N Z J Surg 1992; 62: 385-389.

9. Kitchen PRB. Pilonidal sinus: experience with the Karydakis flap. Br J Surg 1996; 83: 1452-1455.

10. Bannura G, Barrera A, Melo C, Contreras J, Soto D, Mansilla JA. Operación de Karydakis en el tratamiento de la enfermedad pilonidal sacrococcígea. Rev Chil Cir 2005; 57: 340-344.

11. Anderson JH, Yip CO, Nagabhushan JS, Connelly SJ. Day-case Karydakis flap for pilonidal sinus. Dis Colon 
Rectum 2008; 51: 134-138.

12. Bannura G, Cumsille MA, Contreras J, Barrera A, Soto D, Melo C, y cols. Obesidad e hirsutismo como factores predisponentes de la enfermedad pilonidal sacrococcígea. Rev Chil Cir 2007; 59: 136-141.

13. Rodríguez E, Contreras J. Quiste pilonidal: nuestra experiencia con el método cerrado. Rev Chil Cir 1976; 28: 64-68.

14. Vergara JI, Azolas C, Contador J, Pérez-O G, Garrido $\mathrm{R}$, Jensen C. Tratamiento quirúrgico del quiste pilonidal. Rev Chil Cir 1991; 43: 44-46.

15. Jarufe N, Bannura G, Contreras J, Saxton F, Marró P. Enfermedad pilonidal crónica sacrococcígea. Rev Chil Cir 1999; 51: 66-71.

16. Petersen S, Aumann G, Kramer A, Doll D, Sailer M, Hellmich G. Short-term results of Karydakis flap for pilonidal disease. Tech Coloproctol 2007; 11: 235240.

17. Dalenbäck J, Magnusson O, Wedel N, Rimbäck G. Prospective follow-up after ambulatory midline excision of pilonidal sinus and primary suture under local anaesthesia-efficient, sufficient, and persistent. Colorectal Dis 2004; 6: 488-493.

18. Akinci OF, Coskun A, Uzunköy A. Simple and effective surgical treatment of pilonidal sinus. Dis Colon Rectum 2000; 43: 701-707.
19. Cubukcu A, Carkman S, Gonullu NN, Alponat A, Kayabasi B, Eyuboglu E. Lack of evidence that obesity is a cause of pilonidal sinus disease. Eur J Surg 2001; 167: 297-298.

20. Sondenaa K, Andersen E, Nesvik I, Soreide JA. Patient characteristics and symptoms in chronic pilonidal sinus disease. Int J Colorectal Dis 1995; 82: 752-753.

21. Mentes O, Bagci M, Bilgin T, Coskun I, Ozgul O, Ozdemir M. Management of pilonidal sinus disease with oblique excision and primary closure: results of 493 patients. Dis Colon Rectum 2006; 49: 104-108.

22. Gurer A, Gomceli I, Ozgodan M, Ozlem N, Sozen S, Aydin R. Is routine cavity drainage necessary in Karydakis flap operation? A prospective, randomized trial. Dis Colon Rectum 2005; 48: 1797-1799.

23. Aldean I, Shankar PJ, Mathew J, Safarani N, Haboubi Y. Simple excision and primary closure of pilonidal sinus: a simple modification of conventional technique with excellent results. Colorectal Dis 2005; 7: 81-85.

24. Abdul-Ghani AK, Abdul Ghani AN, Ingham Clark. Day care surgery for pilonidal sinus. Ann R Coll Surg Engl 2006; 88: 656-658.

25. Cihan A, Ucan BH, Comert M, Cesur A, Cakmak GK, Tascilar O. Superiority of asymmetric modified Limberg flap for surgical treatment of pilonidal disease. Dis Colon Rectum 2006; 49: 244-249. 